\title{
Hematemesis in a 13-Year-Old Boy due to a Dieulafoy Lesion in His Stomach: A Case Report
}

\author{
Mara Jurić-Kavelj ${ }^{1}$, Ranka Despot ${ }^{2}$, Vanda Žitko², Tanja Kovačević ${ }^{2}$ Tatjana Ćatipović Ardalić ${ }^{2}$, Branka Polićn ${ }^{2,5}$, \\ Damir Aličićc ${ }^{3}$ Dražen Budimir ${ }^{4}$, Joško Markić ${ }^{2,5}$ \\ ${ }^{1}$ Department of Pediatrics, General Hospital Dubrovnik, Dubrovnik, Croatia, ${ }^{2}$ Department of Pediatrics, University Hospital \\ of Split, Split, Croatia, ${ }^{3}$ Division of Gastroenterology and Hepatology, Department for Internal Medicine, University Hospital \\ of Split, Split, Croatia, ${ }^{4}$ Department of Pediatric Surgery, University Hospital of Split, Split, Croatia, ${ }^{5}$ Department of Pediatrics, \\ University of Split School of Medicine, Split, Croatia
}

Correspondence: josko.markic@gmail.com; Tel.: +385 21556 686; Fax.: +38521556590

Received: March 15, 2021; Accepted: June 5, 2021

\begin{abstract}
Objective - Massive gastrointestinal bleeding in children is rare. A Dieulafoy lesion is an uncommon condition which may lead to massive and repeated upper gastrointestinal bleeding. We report a pediatric case of gastric Dieulafoy lesion which was successfully treated surgically, after repeated unsuccessful endoscopic treatment. Case Report - A previously healthy 13-year-old boy presented with hematemesis upon admission to the hospital. He had tachycardia and mild anemia. A gastroscopy revealed erosive gastritis. During the following days, hematemesis continued to reoccur and consequently led to the development of hemorrhagic shock requiring endoscopic treatment with hemoclipping and adrenalin injection. Despite that, hematemesis continued, and it was decided to perform surgical treatment. During open surgery, a tortuous blood vessel was found, suggesting a Dieulafoy lesion. Conclusion - Although rare, a Dieulafoy lesion should be suspected when treating repeating hematemesis in children. Endoscopic treatment is recommended as first-line management, but rarely, in unclear cases, surgery proves to be a better diagnostic and therapeutic option for massive gastrointestinal bleeding.
\end{abstract}

Key Words: Hematemesis - Adolescent - Dieulafoy • Gastroscopy - Operative Surgical Procedure.

\section{Introduction}

A Dieulafoy lesion (DL) is an extremely rare cause of repeated and sometimes massive upper gastrointestinal (GI) hemorrhage in children. The symptoms and signs range from mild anemia, hematemesis and/or melena to hemorrhagic shock (1). A Dieulafoy lesion is a submucosal ectatic arterial lesion associated with a tiny mucosal defect. The exact process, that results in the tortuosity of the superficial artery that erodes through the mucosa and then may bleed, is unknown. The majority of lesions (75-95\%) are in the proximal part of the stomach, particularly within six centimeters of the gastroesophageal junction on the lesser curve (2). However, DL can be found anywhere in gastrointestinal system, as well as in the respiratory system, especially in the bronchial tree (3). The exact incidence of DL in children remains undefined, while it can cause $0.5 \%$ to $14 \%$ of upper gastrointestinal bleedings in adults (4). DL occurs more frequently in boys, and it can occur at any age, starting from birth. Currently, endoscopy is the method of choice for diagnosis and treatment of patients with acute active bleeding (5).

We present the case of 13-year-old boy with repetitive hematemesis due to a Dieulafoy lesion in his stomach. To our knowledge, this is the first pediatric case reported in Croatia, also including neighboring countries. 


\section{Case Report}

A previously healthy 13-year-old boy was admitted to the Department of Pediatrics of Dubrovnik General Hospital because of hematemesis preceded by epigastric pain, without melena or hematochezia. The medical history revealed no previous trauma. He had stopped taking phenoxymethyl penicillin due to tonsillopharyngitis four days earlier. At the time of admission he was conscious but adynamic, pale, and tachycardic, with pulse frequency $117 /$ min and blood pressure 124/79 $\mathrm{mmHg}$. His abdomen was only slightly painful in the epigastrium. Laboratory findings revealed mild anemia, with erythrocyte count of $3.71 \mathrm{~T} / \mathrm{L}$, hemoglobin level of $106 \mathrm{~g} / \mathrm{L}$, hematocrit of $0.311 \mathrm{~L} / \mathrm{L}$, mean corpuscular volume of $83.8 \mathrm{fL}$ and platelets of $341 \mathrm{G} / \mathrm{L}$. His urea level was increased to $10.2 \mathrm{mmol} / \mathrm{L}$, with normal creatinine $(58 \mu \mathrm{mol} / \mathrm{L})$. The coagulation tests were normal. Abdominal ultrasound and chest X-ray were unremarkable. He was given crystalloids and proton pump inhibitor pantoprazole, intravenously. Oral intake was omitted and a nasogastric tube was placed. During the subsequent 24-hours he remained stable, without vomiting and/or signs of bleeding from the nasogastric tube. After the laboratory results showed a significant drop in red blood cell (RBC) count (erythrocytes 3.07 T/L, hemoglobin $89 \mathrm{~g} / \mathrm{L}$ and hematocrit $0.256 \mathrm{~L} / \mathrm{L}$ ), a $\mathrm{RBC}$ transfusion was ordered. Due to the inability to perform endoscopic examination, the boy was referred to the Pediatric Intensive Care Unit of the University Hospital in Split.

Upon admission, a gastroscopy was performed revealing erosive gastritis, but not requiring more aggressive treatment. A test for Helicobacter pylori was negative. He continued to receive proton pump inhibitors intravenously, together with parenteral nutrition. However, three days later, massive hematemesis occurred, leading to a hemorrhagic shock. Boluses of crystalloids, octreotide, tranexamic acid, vitamin $\mathrm{K}$, human prothrombin complex, red blood cell transfusion and fresh frozen plasma were administered. Upon stabilization, a second gastroscopy was performed. A deep ulcer was found, $0.5 \mathrm{~cm}$ in diameter with a bleeding bot- tom, in the corpus of the stomach. In order to stop the bleeding, diluted adrenaline was injected and three clips were inserted, followed by continuous octreotide infusion and high-dose proton pump inhibitors. On the basis of the laboratory results showing anemia, RBC transfusions were repeated, together with fresh frozen plasma and platelets. Gradually, over the next four days, the boy's general condition and laboratory results improved. Slowly, oral intake was initiated. Unfortunately, four days after that, another massive hematemesis occurred, causing hemorrhagic shock with an erythrocyte count of $2.24 \mathrm{~T} / \mathrm{L}$, hemoglobin $66 \mathrm{~g} / \mathrm{L}$ and hematocrit $0.19 \mathrm{~L} / \mathrm{L}$. Besides the therapy for shock, a continuous 48-hour proton pump inhibitor infusion was administered. The third gastroscopy showed a coagulum with previously inserted clips around it in the subcardial part of the stomach, on a lesser curve. The attempt to set up a new clip was unsuccessful, and persistent bleeding was seen. Diluted adrenalin was applied to slow down the bleeding, and another gastroscopy was scheduled for the following day. One clip was removed and two additional clips were placed to secure hemostasis. At that moment, due to the inconsistent endoscopy findings, we suspected a vascular anomaly. A digital subtraction angiography of the visceral arteries was performed and it was unremarkable. In the following days the patient was in a good general condition, without vomiting. Conservative therapy was continued.

On the $21^{\text {th }}$ day of treatment there was another massive hematemesis with signs of hemorrhagic shock, requiring intensive treatment of the shock, including RBC transfusion. At that moment, surgical treatment was indicated. Upper midline laparotomy and explorative gastrotomy were performed. On the lesser curve of the stomach, after the removal of several metal clips, a tortuous submucosal blood vessel with several active bleeding spots was identified, consistent with a Dieulafoy lesion. Bleeding was stopped with $\mathrm{Z}$ ligatures. Early postoperative recovery was without complications. On the $14^{\text {th }}$ postoperative day, the boy was discharged home from the hospital. During the 15-month- 
long follow-up by a pediatric gastroenterologist, no more bleeding incidents were observed.

\section{Discussion}

The diagnosis of DL is often delayed, even after repeated upper gastrointestinal bleedings and endoscopic examinations. Before the widespread use of endoscopy, this condition was diagnosed during surgery or autopsy (6). DL is usually located in the fundus or in the lesser curve of the stomach (3). Recently, endoscopy has become the method of choice, both for diagnosis and for treatment of gastrointestinal bleeding (5). It enables several techniques to be used, such as adrenalin injections, as well as mechanical devices such as clips or banding insertions. These procedures have been generally effective, even in children (7). The best therapeutic choice for gastrointestinal bleeding depends on the clinical presentation, the site of the lesion, and the physician's own expertise in the field (8). Before 1990 , hemorrhage caused by DL was treated surgically (9), but in the following years endoscopic treatment was recommended as the first-line management (10). Even though endoscopy is also the first diagnostic tool, it has only a $70 \%$ diagnostic yield in the diagnosis of DL due to the often small lesions or lesions covered by an adherent clot (11). In a retrospective study of 177 patients in whom DL was a cause of GI bleeding, the first endoscopy did not reliably detect the bleeding source in $33 \%$ of cases, so repeated endoscopies were performed. If endoscopic therapy is unsuccessful, therapeutic alternatives are surgical intervention or angiography with embolization (12).

In this case report we corroborate previous findings regarding the diagnosis and usual location of $\mathrm{DL}$ on the lesser curve of the stomach. Also, we confirm the necessity for repeated endoscopies in some patients, as well as an alternative therapeutic treatment if endoscopic therapy fails (12). Additionally, surgical intervention might not just be a definitive treatment, it might also be a road map to diagnosis.

\section{Conclusion}

Although endoscopic treatment is recommended as the first-line management of gastrointestinal bleeding, in rare, repetitive and unclear cases, surgery proves to be a better diagnostic and therapeutic option. At the moment, there is no consensus regarding the optimum treatment of DL. However, DL should be definitely suspected when treating repeating hematemesis in children.

Authors' Contributions: Conception and design: MJK, JM; Acquisition, analysis and interpretation of data: $\mathrm{MJK}, \mathrm{RD}$, VŽ, TK, TĆA, BP, DA, DB, JM; Drafting the article: MJK, JM; Revising it critically for important intellectual content: BP, TK, JM; Approved final version of the manuscript: MJK, RD, VŽ, TK, TĆA, BP, DA, DB, JM.

Conflict of Interest: The authors declare that they have no conflict of interest.

\section{References}

1. Ünal F, Çakır M, Baran M, Duygulu Ş, Aydoğdu S. Application of endoscopic hemoclips for nonvariceal upper gastrointestinal bleeding in children. Turk J Gastroenterol. 2014;25:147-51.

2. Apiratpracha W, Ho JK, Powell JJ, Yoshida EM. Acute lower gastrointestinal bleeding from a dieulafoy lesion proximal to the anorectal junction post-orthotopic liver transplant. World J Gastroenterol. 2006;12(46):7547-8.

3. Senger JL, Kanthan R. The evolution of Dieulafoy's lesion since 1897: then and now-a journey through the lens of a pediatric lesion with literature review. Gastroenterol Res Pract. 2012;2012:432517.

4. Lim W, Kim TO, Park SB, Rhee HR, Park JH, Bae JH, et al. Endoscopic Treatment of Dieulafoy Lesions and Risk Factors for Rebleeding. Kor J Intern Med. 2009;24(4):318.

5. Itani M, Alsaied T, Charafeddine L, Yazbeck N. Dieulafoy's lesion in children. J Pediatr Gastroenterol Nutr. 2010;51:672-4.

6. Raju GS, Gerson L, Das A, Lewis B, American Gastroenterological Association. American Gastroenterological Association (AGA) Institute medical position statement on obscure gastrointestinal bleeding. Gastroenterology. 2007;133(5):1694-6.

7. Nojkov B, Cappell MS. Gastrointestinal bleeding from Dieulafoy's lesion: Clinical presentation, endoscopic findings, and endoscopic therapy. World J Gastrointest Endosc. 2015;7(4):295-307. 
8. Di Nardo G, Esposito G, Mauro A, Zenzeri L, Ciccarelli GP, Catzola A, et al. Dieulafoy lesion: two pediatric case reports. Ital J Pediatr. 2020;46(1):48.

9. Reilly HF, Al-kawas FH. Dieulafoy's lesion-diagnosis and management. Dig Dis Sci. 1991;36:1702-7.

10. Jeon HK, Kim GH. Endoscopic Management of Dieulafoy's lesion. Clin Endosc. 2015;48:112-20.
11. Chung YF, Wong WK, Soo KC. Diagnostic failures in endoscopy for acute upper gastrointestinal haemorrhage. $\mathrm{Br}$ J Surg. 2000;87(5):614-7.

12. Reilly HF 3rd, al-Kawas FH. Dieulafoy's lesion. Diagnosis and management. Dig Dis Sci. 1991;36(12):1702-7. 